\title{
A VARIATIONAL PROBLEM RELATED TO AN OPTIMAL FILTER PROBLEM WITH SELF-CORRELATED NOISE
}

\author{
BY \\ LEONARD D. BERKOVITZ( $\left.{ }^{(}\right)$AND HARRY POLLARD
}

1. Introduction. In this paper we solve a variational problem generated by an optimal filter problem with correlated noise. The solution of the variational problem will furnish the solution to the filter problem. The variational problem, which we call the " $G$-problem," is the following.

Problem (G-Problem). Let $Y$ denote the class of functions $y$ that are in $L_{1}[0, \infty]$, that are absolutely continuous, that satisfy

$$
y(0)=0
$$

and have the property that the function $G$ defined by

$$
G(u)=-y^{\prime}(u)+e^{-u}+e^{-u} \int_{0}^{u} e^{t} y^{\prime}(t) d t
$$

is in $L_{2}[0, \infty]$. Minimize the functional

$$
J(y)=\left(\int_{0}^{\infty}|y| d t\right)^{2}+\int_{0}^{\infty} G^{2} d t
$$

in the class $Y$.

The filter problem that generates the variational problem will now be described. A precise mathematical formulation of the filter problem and its relation to the variational problem will be given in succeeding sections.

Let $\Phi$ denote the class of functions $f$ of class $C^{(2)}$ on $(-\infty, \infty)$ such that $\left|f^{\prime \prime}(t)\right| \leqq 1$ for all $t$. A function $f$ in $\Phi$ represents an incoming signal. This signal is accompanied by a "noise" $g$. The problem is to filter out the noise for the whole class $\Phi$ in an optimal fashion using the following criterion of optimality. If the function $K$ represents the filter, then at time $t$ the deviation between the actual signal $f(t)$ and the filtered signal is given by

$$
\Psi(t)=f(t)-\int_{0}^{\infty} K(u)[f(t-u)+g(t-u)] d u .
$$

Let $E$ denote the expectation operator, let $N$ denote the family of noise sample functions, and let $m$ be defined by

$$
m=\inf _{K} \sup _{t \geqq 0} \sup _{\Phi} E_{N}\left[(\Psi(t))^{2}\right]
$$

Received by the editors August 19, 1968 and, in revised form, December 18, 1968.

( ${ }^{1}$ ) The work of this author was supported by NSF Grant GP-7372. 
If $m$ is finite, we wish to determine a filter $K^{*}$ that achieves the value $m$. A precise mathematical formulation will be given below.

The present criterion for the optimality of the filter is different from the criterion of Wiener [1]. Our criterion is a reasonable one in problems such as the following tracking problem. The tracked object is permitted to maneuver in a fashion not known in advance by the tracker. The only information available to the tracker is the bound on the absolute value of the acceleration of the tracked object. The tracker wishes to guarantee that the maximum deviation (averaged over all noise sample functions) of the actual position of the tracked object from the position read by the tracking device does not exceed a given error.

In [2] we considered this problem, assuming "white" noise. We solved a general variational problem whose solution in a certain special case furnished the solution of the optimal filter problem. In this paper we shall consider correlated noise, using the special correlation function $\exp (-|x|) / 2$. For treating correlated noise it will be necessary to consider filters that are Borel measures. It will turn out, however, that for our particular problem the optimal filter is the measure resulting from a function of bounded variation with precisely one jump at the origin.

2. Reduction of filter problem to a variational problem. It is well known (see e.g. $[3$, Chapter 8]) that to every bounded Borel measure $\mu$ on $[0, \infty)$ there corresponds a function $y^{\prime}$ of bounded variation on $[0, \infty)$ and left continuous, such that

$$
y^{\prime}(u)=\mu([u, \infty))=\int_{u}^{\infty} d \mu
$$

and such that

$$
\lim _{u \rightarrow \infty} y^{\prime}(u)=0 \text {. }
$$

Conversely, to every left-continuous function $y^{\prime}$ of bounded variation on $[0, \infty)$ and satisfying (2.2) there corresponds a Borel measure $\mu$ related to $y^{\prime}$ by (2.1). We shall, as customary, write the integral of a function $f$ with respect to the measure $\mu$ over a set $A$ as $\int_{A} f d y^{\prime}$ and we shall use the notation $\int_{A} f\left|d y^{\prime}\right|$ to denote the integral of $f$ with respect to the corresponding total variation measure.

Let $C$ be an even, continuous and nonnegative function on $(-\infty, \infty)$. The function $C$ will be the correlation function for the noise.

DEFINITION 1. Let $\Gamma$ denote the class of functions $y^{\prime}$ that are of bounded variation on $[0, \infty)$, are left continuous, satisfy (2.2), satisfy

$$
\int_{0}^{\infty} u^{2}\left|d y^{\prime}\right|<\infty
$$

and satisfy

$$
\int_{0}^{\infty} \int_{0}^{\infty} C(v-u)\left|d y^{\prime}(u)\right|\left|d y^{\prime}(v)\right|<\infty
$$


Since (2.3) holds and since

$$
\int_{u}^{\infty} \tau^{2}\left|d y^{\prime}\right| \geqq u^{2} \int_{u}^{\infty}\left|d y^{\prime}\right|
$$

we get

$$
\left|y^{\prime}(u)\right|=\left|\int_{u}^{\infty} d y^{\prime}\right|<\int_{u}^{\infty}\left|d y^{\prime}\right|=o\left(u^{-2}\right)
$$

Hence $y^{\prime}$ is in $L_{1}[0, \infty)$, and the function $y$ defined by

$$
y(u)=-\int_{u}^{\infty} y^{\prime}(t) d t
$$

satisfies the relation

$$
y(u)=o\left(u^{-1}\right) .
$$

From (2.4) and Fubini's theorem we get

$$
\begin{aligned}
\int_{0}^{\infty} \int_{0}^{\infty} C(v-u) d y^{\prime}(u) d y^{\prime}(v) & =\int_{0}^{\infty} d y^{\prime}(v) \int_{0}^{\infty} C(v-u) d y^{\prime}(u) \\
& =\int_{0}^{\infty} d y^{\prime}(u) \int_{0}^{\infty} C(v-u) d y^{\prime}(v) .
\end{aligned}
$$

We now formulate the filter problem. Let $\Phi$ denote the class of functions $f$ of class $C^{(2)}$ on $(-\infty, \infty)$ such that $\left|f^{\prime \prime}(t)\right| \leqq 1$ for all $t$. Let $N$ denote the family of noise sample functions such that

$$
E_{N}(g)=0, \quad E_{N}(g(t) g(t-\tau))=C(\tau),
$$

where $E$ denotes the expectation operator. Let

$$
\Psi(t)=f(t)+\int_{0}^{\infty} f(t-u) d y^{\prime}(u)+\int_{0}^{\infty} g(t-u) d y^{\prime}(u)
$$

where $y^{\prime}$ is in $\Gamma$. Note that our filter is now a Borel measure, $-d y^{\prime}$. Let

$$
m=\inf _{\Gamma} \sup _{t \geqq 0} \sup _{\Phi} E_{N}\left[\Psi(t)^{2}\right] .
$$

Filter Problem. Determine whether $m$ is finite. If $m$ is finite, determine whether there exists $a y^{\prime}$ in $\Gamma$ for which $m$ is attained. If such a $y^{\prime}$ exists, and is unique, find it.

We now transform this problem into a variational problem. It follows from the definition of $\Psi$, from (2.8), and from (2.9) that

$$
E_{N}\left[(\Psi(t))^{2}\right]=\left[f(t)+\int_{0}^{\infty} f(t-u) d y^{\prime}(u)\right]^{2}+\int_{0}^{\infty} \int_{0}^{\infty} C(v-u) d y^{\prime}(u) d y^{\prime}(v)
$$

Since $\left|f^{\prime \prime}(u)\right| \leqq 1$ we have $f^{\prime}(u)=O(|u|)$ and. $f(u)=O\left(u^{2}\right)$. From this and from (2.3) it follows that the first integral on the right in (2.10) exists. Since $f$ is of class $C^{(2)}$ 
and $y^{\prime}$ is in $L_{1}[0, \infty]$, we may integrate by parts twice and use the relations $f(u)$ $=O\left(u^{2}\right), f^{\prime}(u)=O(|u|),(2.5),(2.6)$ and (2.7) to get the relation

$$
\int_{0}^{\infty} f(t-u) d y^{\prime}(u)=-y^{\prime}(0) f(t)+f^{\prime}(t) y(0)+\int_{0}^{\infty} f^{\prime \prime}(t-u) y(u) d u .
$$

If we substitute this expression into (2.10) we get

$$
\begin{aligned}
E_{N}\left[(\Psi(t))^{2}\right]= & {\left[\left(1-y^{\prime}(0)\right) f(t)+f^{\prime}(t) y(0)+\int_{0}^{\infty} f^{\prime \prime}(t-u) y(u) d u\right]^{2} } \\
& +\int_{0}^{\infty} \int_{0}^{\infty} C(v-u) d y^{\prime}(u) d y^{\prime}(v) .
\end{aligned}
$$

Since we can always add a linear function to $f$ without leaving the class $\Phi$ or altering the value of the integral

$$
\int_{0}^{\infty} f^{\prime \prime}(t-u) y(u) d u
$$

it follows that $E_{n}\left[(\Psi(t))^{2}\right]$ cannot have a finite supremum over $\Phi$ unless

$$
y^{\prime}(0)=1, \quad y(0)=0 .
$$

If we impose (2.11) we get

$$
m=\inf _{\Gamma^{\prime}} \sup _{t \geqq 0} \sup _{\Phi}\left[\left(\int_{0}^{\infty} f^{\prime \prime}(t-u) y(u) d u\right)^{2}+\int_{0}^{\infty} \int_{0}^{\infty} C(v-u) d y^{\prime}(u) d y^{\prime}(v)\right]
$$

where the infimum is taken on the subset $\Gamma^{\prime}$ of $\Gamma$ defined by (2.11). Since

$$
\sup _{\left|f^{\prime \prime}\right| \leqq 1} \int_{0}^{\infty} f^{\prime \prime}(t-u) y(u) d u=\int_{0}^{\infty}|y(u)| d u
$$

we finally get

$$
m=\inf _{\Gamma^{\prime}}\left\{\left(\int_{0}^{\infty}|y| d u\right)^{2}+\int_{0}^{\infty} \int_{0}^{\infty} C(u-v) d y^{\prime}(u) d y^{\prime}(v)\right\}
$$

Thus, we have reduced the filter problem to the following variational problem.

Problem 1. Minimize the functional

$$
J_{1}(y)=\left(\int_{0}^{\infty}|y| d u\right)^{2}+\int_{0}^{\infty} \int_{0}^{\infty} C(u-v) d y^{\prime}(u) d y^{\prime}(v)
$$

in the class $A$ of functions $y$ that are absolutely continuous on $[0, \infty)$, that are in $L_{1}[0, \infty]$, that satisfy $(2.11)$, and possess derivatives $y^{\prime}$ such that $y^{\prime}$ belongs to $\Gamma$.

In [2], where we treat the case of "white" noise, $C$ is the delta function and the functions $y^{\prime}$ are taken to be absolutely continuous. The variational problem obtained there differs from this problem in that the double integral in (2.12) was replaced by $\int_{0}^{\infty}\left(y^{\prime \prime}\right)^{2} d u$. The reader may find it instructive to compare the arguments and results of this paper with those of [2]. 
3. The $G$-problem. In this section we formulate a problem related to Problem 1 for the case in which

$$
C(u)=e^{-|u|} / 2 .
$$

The new problem is obtained by transforming the double integral in (2.12).

Define

$$
G(u)=-2 \int_{0}^{u} C(u-v) d y^{\prime}(v)=-e^{-u} \int_{0}^{u} e^{v} d y^{\prime}(v) .
$$

Upon integrating by parts and using $y^{\prime}(0)=1$, we get

$$
G(u)=-y^{\prime}(u)+e^{-u}+e^{-u} \int_{0}^{u} e^{v} y^{\prime}(v) d v .
$$

Clearly, $G$ is measurable and $G(u)$ is finite for all $u \geqq 0$. Therefore, we may consider

$$
\begin{aligned}
\int_{0}^{\infty} G^{2}(u) d u & =2 \int_{0}^{\infty}|G(u)|\left|\int_{0}^{u} C(u-w) d y^{\prime}(w)\right| d u \\
& \leqq 2 \int_{0}^{\infty}|G(u)|\left(\int_{0}^{u} C(u-w)\left|d y^{\prime}(w)\right|\right) d u \\
& =2 \int_{0}^{\infty}\left|d y^{\prime}(w)\right| \int_{w}^{\infty}|G(u)| C(u-w) d u \\
& =4 \int_{0}^{\infty}\left|d y^{\prime}(w)\right| \int_{w}^{\infty} C(u-w) d u\left|\int_{0}^{u} C(u-v) d y^{\prime}(v)\right| \\
& \leqq 4 \int_{0}^{\infty}\left|d y^{\prime}(w)\right| \int_{w}^{\infty} C(u-w) d u \int_{0}^{u} C(u-v)\left|d y^{\prime}(v)\right|
\end{aligned}
$$

Since $u \geqq v$ and $u \geqq w$, we may write

$$
\int_{0}^{u} C(u-v)\left|d y^{\prime}(v)\right|=\int_{0}^{w} C(u-v)\left|d y^{\prime}(v)\right|+\int_{w+0}^{u} C(u-v)\left|d y^{\prime}(v)\right| .
$$

We remind the reader of the explanation of notation given in the first paragraph of $\S 2$. If we substitute $\left(3.4^{1}\right)$ into the last member of (3.4) we get

$$
\int_{0}^{\infty} G^{2}(u) d u \leqq I_{1}+I_{2}
$$

where

$$
\begin{aligned}
& I_{1}=4 \int_{0}^{\infty}\left|d y^{\prime}(w)\right| \int_{w}^{\infty} C(u-w) d u \int_{0}^{w} C(u-v)\left|d y^{\prime}(v)\right| \\
& I_{2}=4 \int_{0}^{\infty}\left|d y^{\prime}(w)\right| \int_{w}^{\infty} C(u-w) d u \int_{w+0}^{u} C(u-v)\left|d y^{\prime}(v)\right| .
\end{aligned}
$$

We shall show that the integrals $I_{1}$ and $I_{2}$ are finite. It will then follow from Fubini's theorem and the chain of inequalities (3.4) that we can remove the absolute value signs throughout (3.4) and in the integrals $I_{1}$ and $I_{2}$ and replace 
the inequality signs by equal signs. In particular, equality will hold in (3.5) with the absolute value removed from the integrals $I_{1}$ and $I_{2}$.

In $I_{1}$ we interchange the order of integration in the last two integrals to get

$$
I_{1}=4 \int_{0}^{\infty}\left|d y^{\prime}(w)\right| \int_{0}^{w}\left|d y^{\prime}(v)\right| \int_{w}^{\infty} C(u-v) C(u-w) d u .
$$

If we use (3.1) and recall that $u \geqq v, u \geqq w$, we obtain on integrating that

$$
I_{1}=\frac{1}{2} \int_{0}^{\infty} e^{-w}\left|d y^{\prime}(w)\right| \int_{0}^{w} e^{v}\left|d y^{\prime}(v)\right|
$$

The right-hand side is finite by virtue of (2.4).

In $I_{2}$ we also interchange the order of integration and get

$$
I_{2}=4 \int_{0}^{\infty}\left|d y^{\prime}(w)\right| \int_{w+0}^{\infty}\left|d y^{\prime}(v)\right| \int_{v}^{\infty} C(u-v) C(u-w) d u,
$$

which is readily seen to yield

$$
I_{2}=\frac{1}{2} \int_{0}^{\infty} e^{w}\left|d y^{\prime}(w)\right| \int_{w+0}^{\infty} e^{-v}\left|d y^{\prime}(v)\right| .
$$

The right-hand side is finite by virtue of (2.4).

Since $I_{1}$ and $I_{2}$ are finite we may remove the absolute values from $I_{1}$ and $I_{2}$ in (3.5) and equality will hold in the resulting relation. Moreover, from the arguments in the two preceding paragraphs and from Fubini's theorem it follows that if we remove the absolute values from $I_{1}$ and $I_{2}$, then the resulting integrals are given by the right-hand sides of (3.6) and (3.7) with the absolute values removed. Hence we conclude that

$$
\int_{0}^{\infty} G^{2}(u) d u=\frac{1}{2} \int_{0}^{\infty} e^{-w} d y^{\prime}(w) \int_{0}^{w} e^{v} d y^{\prime}(v)+\frac{1}{2} \int_{0}^{\infty} e^{w} d y^{\prime}(w) \int_{w+0}^{\infty} e^{-v} d y^{\prime}(v) .
$$

Hence

$$
\int_{0}^{\infty} G^{2}(u) d u=\frac{1}{2} \int_{0}^{\infty} d y^{\prime}(w) \int_{0}^{\infty} e^{-|w-v|} d y^{\prime}(v)
$$

Using (2.8) we finally get

$$
\begin{aligned}
\int_{0}^{\infty} G^{2}(u) d u & =\frac{1}{2} \int_{0}^{\infty} \int_{0}^{\infty} e^{-|w-v|} d y^{\prime}(v) d y^{\prime}(w) \\
& =\int_{0}^{\infty} \int_{0}^{\infty} C(w-v) d y^{\prime}(w) d y^{\prime}(v) .
\end{aligned}
$$

Thus, $G$ is in $L_{2}[0, \infty]$ and we may write $(2.12)$ as follows:

$$
J_{1}(y)=\left(\int_{0}^{\infty}|y| d u\right)^{2}+\int_{0}^{\infty} G^{2} d u .
$$

These considerations suggest the $G$-problem formulated in the introduction. 
Let $Y_{\Gamma}$ be the subset of $Y$ consisting of functions $y$ whose derivatives $y^{\prime}$ are in $\Gamma$. The discussion that led to formula (3.8) shows that the class $A$ of Problem 1 is a subclass of $Y_{\Gamma}$. Hence $A \subset Y_{\Gamma} \subset Y$ and so $Y_{\Gamma}$ and $Y$ are not void. The discussion that led to formula (3.8) also shows that if $y$ is in $A$ then

$$
J_{1}(y)=J(y) .
$$

The formulation of the $G$-problem was suggested by the filter problem and Problem 1. Assuming for the moment that the $G$-problem has a solution $z$, it is not a priori clear that $z$ will furnish a solution to the filter problem. If, however, it turns out that $z^{\prime}$ is in $\Gamma$, then $z$ will furnish a solution to Problem 1 and to the filter problem in the following manner.

Define

$$
\begin{aligned}
& \zeta^{\prime}(u)=z^{\prime}(u), \quad u>0, \\
& \zeta^{\prime}(0)=1, \\
& \zeta(u)=\int_{0}^{u} \zeta^{\prime} d t .
\end{aligned}
$$

Then $\zeta(u)=z(u)$ for all $u, \zeta$ satisfies (2.11) and $\zeta^{\prime}$ is in $\Gamma$. Hence $\zeta$ is in $A$. Therefore, by (3.9)

$$
J(\zeta)=J_{1}(\zeta)
$$

On the other hand, $J(\zeta)=J(z) \leqq J(y)$ for all $y$ in $Y$, and so again by (3.9) and (3.11)

$$
J_{1}(\zeta) \leqq J_{1}(y) \text { for all } y \text { in } A \text {. }
$$

But this says that $\zeta$ is a solution to Problem 1. Hence $\zeta^{\prime}$ is a solution of the original filter problem.

We shall show that the $G$-problem has a unique solution $z$ and we shall find this solution. From the form of the solution it will be clear that $z$ is in $Y_{\Gamma}$. Moreover, we shall show that the solution $z$ has an absolutely continuous derivative $z^{\prime}$. Hence the function $\zeta^{\prime}$ defined in (3.10) is unique, except perhaps at the origin. At the origin, however, we must have $\zeta^{\prime}(0)=1$ for $\zeta^{\prime}$ to be a solution of the filter problem. Hence $\zeta^{\prime}$ is unique, and the filter problem has a unique solution.

4. Existence and uniqueness of solution to $G$-problem. In this section we prove the following result.

THEOREM 1. The G-problem has a unique solution.

We begin the proof by obtaining a second relation between $y$ and $G$ that is equivalent to (1.2). For convenience we shall call functions $y$ and $G$ that are related by (1.2), related functions. If we define

$$
G^{(1)}(v)=\int_{0}^{v} G d t
$$


then it follows from (1.2) and (1.1) by straightforward integration that

$$
G^{(1)}(v)=1-e^{-v}-e^{-v} \int_{0}^{v} e^{t} y^{\prime}(t) d t=1-y^{\prime}(v)-G(v) .
$$

Further integration from 0 to $u$ and the use of (1.1) yields

$$
y(u)=u-G^{(1)}(u)-G^{(2)}(u),
$$

where $G^{(2)}(u)=\int_{0}^{u} G^{(1)} d t$.

Equation (4.1) is the desired relation. To show its equivalence to (1.2) we start with (4.1) and differentiate both sides to get

$$
-y^{\prime}(u)+1=G(u)+G^{(1)}(u) .
$$

Hence

$$
-e^{u}\left(y^{\prime}(u)-1\right)=e^{u}\left(G(u)+G^{(1)}(u)\right)=\left(e^{u} G^{(1)}(u)\right)^{\prime} .
$$

Integration gives

$$
G^{(1)}(u)=1-e^{-u}-e^{-u} \int_{0}^{u} e^{t} y^{\prime}(t) d t .
$$

If we substituce this into (4.2) we get (1.2).

Let $m=\inf [J(y): y \in Y]$. Then $m \geqq 0$ and there exists a sequence of functions $y_{n}$ in $Y$ such that $\lim _{n \rightarrow \infty} J\left(y_{n}\right)=m$. Hence there exists a constant $M>0$ such that

$$
\int_{0}^{\infty}\left|y_{n}\right| d t \leqq M, \quad \int_{0}^{\infty}\left(G_{n}\right)^{2} d t \leqq M
$$

where $G_{n}$ is related to $y_{n}$. From the second inequality in (4.3) and the weak compactness of closed balls in $L_{2}$ it follows that there exists an element $F$ in $L_{2}[0, \infty]$ and a subsequence of the sequence $\left\{y_{n}\right\}$, which we relabel as $\left\{y_{n}\right\}$, such that

$$
G_{n} \rightarrow F \quad \text { weakly in } L_{2}[0, \infty]
$$

Define

$$
\begin{aligned}
z(u) & =u-\int_{0}^{u} F(t) d t-\int_{0}^{u}(u-t) F(t) d t \\
& =u-F^{(1)}(u)-F^{(2)}(u)
\end{aligned}
$$

where $F^{(i)}, i=1,2$, is the $i$ th integral of $F$ with lower limit of integration taken as zero. From (4.1) and the definition of $G^{(1)}$ and $G^{(2)}$ we have

$$
y_{n}(u)=u-\int_{0}^{u} G_{n}(t) d t-\int_{0}^{u}(u-t) G_{n}(t) d t .
$$

It now follows from (4.4) that for all $u \geqq 0$,

$$
\lim _{n \rightarrow \infty} y_{n}(u)=z(u)
$$


Hence by Fatou's Lemma and (4.3) we get that

$$
\int_{0}^{\infty}|z| d t \leqq \liminf _{n \rightarrow \infty} \int_{0}^{\infty}\left|y_{n}\right| d t \leqq M .
$$

Therefore, $z$ is in $L_{1}[0, \infty]$. It is clear from (4.5) that $z$ is absolutely continuous and that $z(0)=0$. From (4.5) and the equivalence of (4.1) and (1.2) we get that $F$ and $z^{\prime}$ are related by (1.2). Hence since $F$ is in $L_{2}[0, \infty]$, the function $z$ is in $Y$.

We now show that $z$ is a minimizing element; i.e. that $J(z) \leqq J(y)$ for all $y$ in $Y$. Since $F$ is the weak limit of $G_{n}$ and since

$$
\int_{0}^{\infty} G_{n} F d t \leqq\left(\int_{0}^{\infty} G_{n}^{2} d t\right)^{1 / 2}\left(\int_{0}^{\infty} F^{2} d t\right)^{1 / 2},
$$

we get that

$$
\int_{0}^{\infty} F^{2} d t \leqq\left(\int_{0}^{\infty} F^{2} d t\right)^{1 / 2} \liminf _{n \rightarrow \infty}\left(\int_{0}^{\infty} G_{n}^{2} d t\right)^{1 / 2}
$$

Therefore,

$$
\int_{0}^{\infty} F^{2} d t \leqq \liminf _{n \rightarrow \infty} \int_{0}^{\infty} G_{n}^{2} d t
$$

Using this inequality and (4.6) we get

$$
\begin{aligned}
J(z)= & \left(\int_{0}^{\infty}|z| d t\right)^{2}+\int_{0}^{\infty} F^{2} d t \leqq \liminf _{n \rightarrow \infty}\left(\int_{0}^{\infty}\left|y_{n}\right| d t\right)^{2} \\
& +\liminf _{n \rightarrow \infty} \int_{0}^{\infty} G_{n}^{2} d t=\liminf _{n \rightarrow \infty}\left[\left(\int_{0}^{\infty}\left|y_{n}\right| d t\right)^{2}+\int_{0}^{\infty} G_{n}^{2} d t\right] \\
= & \lim _{n \rightarrow \infty} J\left(y_{n}\right)=m .
\end{aligned}
$$

On the other hand, since $z$ is in $Y$, we have $J(z) \geqq m$, and so $J(z)=m$, as asserted.

We next show that the minimizing function $z$ is unique. Our first step is to establish the following lemma.

LemMA 1. Let $x$ and $y$ be two functions in $Y$. Let $\alpha \geqq 0, \beta \geqq 0, \alpha+\beta=1$. Then $\alpha x+\beta y$ is in $Y$ and

$$
J(\alpha x+\beta y)^{1 / 2} \leqq \alpha J(x)^{1 / 2}+\beta J(y)^{1 / 2} .
$$

If $\alpha=0$ or $\beta=0$, then there is nothing to prove. So suppose $\alpha>0$ and $\beta>0$. Let $G$ be the function related to $x$ and let $G^{\prime}$ be the function related to $y$. Let $w=\alpha x+\beta y$. Then, clearly, $w$ is in $L_{1}[0, \infty]$ and $w(0)=0$. If $G^{\prime \prime}$ denotes the function related to $w$ then it follows from (1.2) that $G^{\prime \prime}=\alpha G+\beta G^{\prime}$. Hence $G^{\prime \prime}$ is in $L_{2}[0, \infty]$ and so $w$ is in $Y$.

If we let $\|\cdots\|_{1}$ denote the $L_{1}[0, \infty]$ norm and let $\|\cdots\|_{2}$ denote the $L_{2}[0, \infty]$ norm, then from (1.3) we get

$$
\begin{aligned}
J(w) & =\|w\|_{1}^{2}+\left\|G^{\prime \prime}\right\|_{2}^{2}=\|\alpha x+\beta y\|_{1}^{2}+\left\|\alpha G+\beta G^{\prime}\right\|_{2}^{2} \\
& \leqq\left(\alpha\|x\|_{1}+\beta\|y\|_{1}\right)^{2}+\left(\alpha\|G\|_{2}+\beta\left\|G^{\prime}\right\|_{2}\right)^{2} .
\end{aligned}
$$


Since $L_{2}$ is completely convex we have that if equality holds then $G^{\prime}=k G$ for some constant $k>0$. Upon squaring and collecting terms on the right we get

$$
J(w) \leqq \alpha^{2} J(x)+\beta^{2} J(y)+2 \alpha \beta\left(\|x\|_{1}\|y\|_{1}+\|G\|_{2}\left\|G^{\prime}\right\|_{2}\right),
$$

where equality implies $G^{\prime}=k G$ for some $k>0$. A straightforward calculation shows that

$$
J(x) J(y)-\left(\|x\|_{1}\|y\|_{1}+\|G\|_{2}\left\|G^{\prime}\right\|_{2}\right)^{2}=\left(\|x\|_{1}\left\|G^{\prime}\right\|_{2}-\|y\|_{1}\|G\|_{2}\right)^{2} .
$$

Thus

$$
J(x)^{1 / 2} J(y)^{1 / 2} \geqq\|x\|_{1}\|y\|_{1}+\|G\|_{2}\left\|G^{\prime}\right\|_{2} .
$$

Substituting this into (4.8) gives

$$
J(w)^{1 / 2} \leqq\left[\alpha J(x)^{1 / 2}+\beta J(y)^{1 / 2}\right],
$$

which is the desired result.

We have also established the following corollary.

COROLlary. If equality holds in (4.7), then $G^{\prime}=k G$ for some $k>0$.

Let us now suppose that there are two minimizing functions $z$ and $z_{0}$ with related functions $F$ and $F_{0}$. Then by Lemma $1,\left(z+z_{0}\right) / 2$ is in $Y$ and

$$
m^{1 / 2} \leqq J\left(\left(z+z_{0}\right) / 2\right)^{1 / 2} \leqq J(z)^{1 / 2} / 2+J\left(z_{0}\right)^{1 / 2} / 2 \leqq m^{1 / 2} .
$$

Hence equality holds throughout, and by the Corollary we get that $F_{0}=k F$ for some $k>0$. Hence, by (4.1)

$$
z(u)=u-F^{(1)}(u)-F^{(2)}(u), \quad z_{0}(u)=u-k F^{(1)}(u)-k F^{(2)}(u) .
$$

If we subtract the second equation from the first we get

$$
z(u)-z_{0}(u)=-(1-k)\left(F^{(1)}(u)+F^{(2)}(u)\right) .
$$

If $k \neq 1$, we can divide through by $(1-k)$ and get that $F^{(1)}+F^{(2)}$ is in $L_{1}[0, \infty]$. But then, $u=z(u)+F^{(1)}(u)+F^{(2)}(u)$ would be in $L_{1}[0, \infty]$. Since this is impossible, we get that $k=1$. Hence $z=z_{0}$ and the uniqueness is established.

\section{Characterization of solution.}

THEOREM 2. Let $z$ be the minimizing function for the G-problem and let $F$ be the corresponding related function. Then $z$ is characterized as follows:

(i) The function $F$ is absolutely continuous and the derivative $z^{\prime}$ is absolutely continuous.

(ii) There exists a finite set of points $0=\alpha_{0}<\alpha_{1}<\cdots<\alpha_{N}=\lambda$ such that $z\left(\alpha_{n}\right)=0$ 
for $n=0,1,2, \ldots, N, z(u)=0$ if $u \geqq \lambda$, and $z(u) \neq 0$ for all other points $u$. On each interval $\left[\alpha_{n}, \alpha_{n+1}\right], n=0,1, \ldots, N-1, z$ is a quartic and for $u$ in $\left(\alpha_{n}, \alpha_{n+1}\right)$

$$
\begin{gathered}
\text { signum } z(u)=(-1)^{n}, \\
z^{(\text {iv })}(u)=(-1)^{n} I,
\end{gathered}
$$

where

$$
I=\int_{0}^{\infty}|z| d u
$$

If $u \geqq \lambda$, then

$$
F(u)=F(\lambda) e^{(\lambda-u)} .
$$

(iii) In each interval $\left[\alpha_{n}, \alpha_{n+1}\right], n=0,1,2, \ldots, N-2$, the derivative $z^{\prime}$ has precisely one zero which occurs at an interior point of the interval $\left(\alpha_{n}, \alpha_{n+1}\right)$. In the last interval $\left[\alpha_{n-1}, \alpha_{n}\right], z^{\prime}$ has precisely one zero in the interior and $z^{\prime}\left(\alpha_{N}\right)=0$.

(iv) At the zeros $\alpha_{1}, \ldots, \alpha_{N-1}$ the right-and left-hand derivatives $z^{\prime \prime}$ and $z^{m}$ satisfy the following relations

$$
\begin{aligned}
z^{\prime \prime}\left(\alpha_{n}+0\right)-z^{\prime \prime}\left(\alpha_{n}-0\right) & =I\left[\sigma\left(\alpha_{n}+0\right)-\sigma\left(\alpha_{n}-0\right)\right] \\
z^{m}\left(\alpha_{n}+0\right) & =z^{m}\left(\alpha_{n}-0\right)
\end{aligned}
$$

where $I$ is defined in (5.3) and $\sigma(u) \equiv$ signum $z(u)$.

(v) The value of the minimum is given by

$$
m=F(0)-F^{2}(0) / 2
$$

and

$$
m=\int_{0}^{\infty} F(t) e^{-t} d t
$$

The proof of this theorem will be given below.

THEOREM 3. The solution of the G-problem furnishes the unique solution to Problem 1 and to the filter problem.

This follows from the discussion at the end of $\S 3$ and the observation that Theorem 2 shows that $z$ is in $Y_{\Gamma}$ and that $z^{\prime}$ is absolutely continuous.

6. Absolute continuity of $F$. In this section we shall prove that $F$ is absolutely continuous. It will then follow from (1.2) with $G$ replaced by $F$ and $y^{\prime}$ replaced by $z^{\prime}$ that $z^{\prime}$ is absolutely continuous. Conclusion (i) of Theorem 2 will then be proved.

The following lemma will be used in the proof of the absolute continuity of $F$.

LEMMA 2. Let $V_{k}$ denote the class of all functions $\eta$ of class $C^{(k-1)}$ on $[0, \infty]$ that: (i) vanish at the origin together with $\eta^{\prime}, \ldots, \eta^{(k-1)}$, (ii) vanish outside of a finite interval, (iii) possess an absolutely continuous $(k-1)$ th derivative. Let $g$ be a function in $L_{1}[0, \infty]$, let I be a positive constant, and for every $\eta$ in $V_{k}$ let

$$
\left|\int_{0}^{\infty} g \eta^{(k)} d t\right| \leqq I \int_{0}^{\infty}|\eta| d t .
$$


Then $g$ is of class $C^{(k-1)}$ on $[0, \infty]$, and $g^{(k-1)}$ is absolutely continuous, and

$$
\left|g^{(k)}(u)\right| \leqq I .
$$

The class $V_{k}$ is clearly a dense subspace of $L_{1}[0, \infty]$. The function $g$ defines a linear functional $\Lambda$ on $V_{k}$ as follows:

$$
\Lambda(\eta)=\int_{0}^{\infty} g \eta^{(k)} d t
$$

From (6.1) it is clear that $\Lambda$ is bounded on $V_{k}$ and $\|\Lambda\|_{k} \leqq I$, where $\|\Lambda\|_{k}$ denotes the norm of $\Lambda$ on $V_{k}$. Hence $\Lambda$ can be extended to all of $L_{1}[0, \infty]$ and the norm $\|\Lambda\|$ of the extended functional will equal $\|\Lambda\|_{k}$. Thus $\|\Lambda\| \leqq I$. Therefore, there exists a function $h$ in $L_{\infty}[0, \infty]$ such that ess sup $|h(u)|=\|\Lambda\| \leqq I$ and

$$
\Lambda(\eta)=\int_{0}^{\infty} g \eta^{(k)} d t=\int_{0}^{\infty} h \eta d t
$$

for every $\eta$ in $V_{k}$. We now integrate by parts $k$ times in the last integral. Since $\eta$ is in $C^{(k-1)}$ and $\eta^{(k-1)}$ is absolutely continuous, and since $\eta, \eta^{\prime}, \ldots, \eta^{(k-1)}$ all vanish at the end points of the intervals on which $\eta \not \equiv 0$, we get the relation

$$
\int_{0}^{\infty}\left[g-H^{(k)}\right] \eta^{(k)} d t=0,
$$

where $H^{(k)}$ is a $k$ th integral of $h$. This relation holds for every $\eta$ in $V_{k}$.

Let us now fix an interval $[a, b]$ and consider all functions $\eta$ in $V_{k}$ that vanish outside of $[a, b]$. We get

$$
\int_{a}^{b}\left[g-H^{(k)}\right] \eta^{(k)} d t=0 .
$$

It now follows from an extension of the fundamental lemma of the calculus of variations (see e.g. $[4$, p. 198]) that almost everywhere on $[a, b]$

$$
g(u)=H^{(k)}(u)+P_{k-1}(u),
$$

where $P_{k-1}$ is a polynomial of degree $k-1$. Clearly, we may redefine $g$ so that the preceding equality holds everywhere. Hence the function $g$ has the asserted properties on $[a, b]$. Since $[a, b]$ is an arbitrary interval, it follows that $g$ has the asserted properties on $[0, \infty]$.

We now prove that $F$ is absolutely continuous. Let $\eta$ be any function in the class $V_{1}$. Then for any real number $\varepsilon$, the function $y_{\varepsilon}=z+\varepsilon \eta$ is absolutely continuous on $[0, \infty]$, and satisfies $y_{\varepsilon}(0)=1$. Here, of course, $z$ is the minimizing function. From (1.2) we see that the function $G_{\varepsilon}$ related to $y_{\varepsilon}$ is given by

$$
G_{\varepsilon}(u)=F(u)+\varepsilon \omega(u),
$$

where

$$
\omega(u)=-\eta^{\prime}(u)+e^{-u} \int_{0}^{u} e^{t} \eta^{\prime}(t) d t
$$


Since $\eta$ is absolutely continuous and vanishes outside of a finite interval we see that $\omega$ is in $L_{2}[0, \infty]$. Hence $G_{\varepsilon}$ is in $L_{2}[0, \infty]$ and so $y_{\varepsilon}$ is in $Y$. Therefore, if we let $m$ denote the minimum of the functional (1.3) we have the following:

$$
\begin{aligned}
m & \leqq\left(\int_{0}^{\infty}|z+\varepsilon \eta|\right)^{2}+\int_{0}^{\infty}(F+\varepsilon \omega)^{2} d t \\
& \leqq m+2|\varepsilon|\left(\int_{0}^{\infty}|z| d t\right)\left(\int_{0}^{\infty}|\eta| d t\right)+|\varepsilon|^{2} \int_{0}^{\infty}|\eta|^{2} d t+2 \varepsilon \int_{0}^{\infty} F \omega d t+\varepsilon^{2} \int_{0}^{\infty} \omega^{2} d t .
\end{aligned}
$$

Since $F, \omega$, and $\eta$ are in $L_{2}[0, \infty]$ and since $z$ and $\eta$ are in $L_{1}[0, \infty]$, all of the integrals on the right are finite.

From the preceding inequality we get

$$
-\varepsilon \int_{0}^{\infty} F \omega d t \leqq|\varepsilon|\left(\int_{0}^{\infty}|z| d t\right)\left(\int_{0}^{\infty}|\eta| d t\right)+\frac{|\varepsilon|^{2}}{2} \int_{0}^{\infty}|\eta|^{2} d t+\frac{\varepsilon^{2}}{2} \int_{0}^{\infty} \omega^{2} d t
$$

If we divide through by positive $\varepsilon$ and then let $\varepsilon$ tend to zero we get

$$
-\int_{0}^{\infty} F \omega d t \leqq I \int_{0}^{\infty}|\eta| d t
$$

where $I$ is defined in (5.3). If, instead, we divide through by negative $\varepsilon$ and then let $\varepsilon$ tend to zero we get

$$
-\int_{0}^{\infty} F \omega d t \geqq-I \int_{0}^{\infty}|\eta| d t
$$

Upon combining the last two displayed inequalities, we get

$$
\left|\int_{0}^{\infty} F \omega d t\right| \leqq I \int_{0}^{\infty}|\eta| d t
$$

If we now substitute the right-hand side of (6.4) into (6.5) and interchange the order of integration in the resulting iterated integral we get

$$
\int_{0}^{\infty} F \omega d t=\int_{0}^{\infty} g \eta^{\prime} d t
$$

where

$$
g(u)=-F(u)+e^{u} \int_{u}^{\infty} F(t) e^{-t} d t .
$$

Since $F$ is in $L_{2}[0, \infty]$, the integral on the right in (6.7) is finite. The interchange of order leading to (6.6) and (6.7) is justified because $F(t)\{\exp (-t)\}$ is in $L_{1}[0, \infty]$ and $\eta$ vanishes outside of a finite interval.

If we now combine (6.6) and (6.5) we get

$$
\left|\int_{0}^{\infty} g \eta^{\prime} d t\right| \leqq I \int_{0}^{\infty}|\eta| d t
$$


Since $\eta$ is an arbitrary element of $V_{1}$ it follows from Lemma 2 that $g$ is absolutely continuous and satisfies (6.2). It then follows from (6.7) that $F$ is absolutely continuous.

The authors acknowledge their thanks to Professor Wendell H. Fleming who suggested the present proof of Lemma 2 to replace a more complicated proof originally given by us.

7. The Euler equation. In this section we deduce a necessary condition that the minimizing function $z$ must satisfy. This condition is the analogue of the Euler equation in classical variational problems.

The minimizing function $z$ is continuous. Therefore, the set $\Omega=\{u: z(u) \neq 0\}$ is open and has a unique representation as the union of an at most countable number of disjoint open intervals $\left\{K_{j}\right\}$.

LEMMA 3. On each component $K_{j}$ of $\Omega$ the function $z$ satisfies the equation

$$
z^{(1 \mathrm{v})}(u)=-I \sigma(u),
$$

where $\sigma(u) \equiv$ signum $z(u)$ and $I$ is given by (5.3). Hence in the closure of each $K$, the function $z$ is a quartic. Moreover, on each component $K_{j}, F$ is a quadratic and

$$
F^{\prime \prime}(u)=I \sigma(u) \text {. }
$$

Since $z$ and $F$ are related functions it follows from (4.1) that

$$
z(u)=u-F^{(2)}(u)-F^{(1)}(u) \text {. }
$$

Therefore, if we establish that $F$ is of class $C^{(2)}$ on components and satisfies (7.2), it will follow by differentiation of (7.3) that (7.1) holds.

Let $(a, b)$ be a component of $\Omega$ and let $c$ and $d$ be real numbers such that $a<c<d$ $<b$. Let $\eta$ be a $C^{(2)}$ function that vanishes outside of $(c, d)$. Then,

$$
\eta(c)=\eta(d)=\eta^{\prime}(c)=\eta^{\prime}(d)=0 .
$$

Also, $\eta$ is in $V_{1}$. For functions $\eta$ in $V_{1}$ we showed in $\S 6$ that for every real $\varepsilon$, the function $z+\varepsilon \eta$ is in $Y$ and the related function $G_{\varepsilon}$ is given by (6.3) and (6.4).

Let

$$
\varphi(\varepsilon)=J(z+\varepsilon \eta)=\left(\int_{0}^{\infty}|z+\varepsilon \eta| d t\right)^{2}+\int_{0}^{\infty}(F+\varepsilon \omega)^{2} d t .
$$

Then, since $z$ minimizes $J(y)$ over $Y$, it follows that the real valued function $\varphi$ has a minimum at $\varepsilon=0$. From the definition of $\eta$ it is clear that for $|\varepsilon|$ sufficiently small,

$$
\operatorname{signum}(z(u)+\varepsilon \eta(u))=\sigma(u) .
$$

Hence, for $|\varepsilon|$ sufficiently small,

$$
\varphi(\varepsilon)=\left[\int_{0}^{c}|z| d t+\int_{c}^{d}(z+\varepsilon \eta) \sigma d t+\int_{d}^{\infty}|z| d t\right]^{2}+\int_{0}^{\infty}(F+\varepsilon \omega)^{2} d t
$$


From (7.5) it is clear that $\varphi^{\prime}$ exists for $|\varepsilon|$ sufficiently small. Since $\varphi$ has a minimum at $\varepsilon=0$, we have $\varphi^{\prime}(0)=0$. If we compute $\varphi^{\prime}(0)$ and set $\varphi^{\prime}(0)=0$, we get

$$
I \int_{c}^{a} \eta \sigma d t+\int_{0}^{\infty} F \omega d t=0
$$

If we now use (6.6) and the definition of $\eta$ we get

$$
I \int_{c}^{d} \sigma \eta d t+\int_{c}^{d} g \eta^{\prime} d t=0
$$

If we set $\sigma_{1}(u)=\int_{c}^{u} \sigma d t=(u-c) \sigma(u), u \in(a, b)$, integrate by parts in the first integral in (7.6) and use (7.4), we get

$$
\int_{c}^{d}\left[-I \sigma_{1}+g\right] \eta^{\prime} d t=0 .
$$

It now follows from the fundamental lemma of the calculus of variations that on $[c, d]$

$$
g(u)=I(u-c) \sigma(u)+A,
$$

where $A$ is a constant. Hence, for $u$ in $[c, d]$

$$
g^{\prime}(u)=I \sigma(u)
$$

Since $c$ and $d$ are arbitrary real numbers satisfying $a<c<d<b$, it follows that (7.7) holds for all $u$ in $(a, b)$.

Upon differentiating (6.7) we get that

$$
g^{\prime}(u)=-F^{\prime}(u)+g(u) \text { a.e. }
$$

Combining (7.7) with (7.8) gives

$$
I \sigma(u)=-F^{\prime}(u)+g(u)
$$

for $u$ in a component $K_{j}$. If necessary, we can redefine $F$ and $F^{\prime}$ on sets of measure zero so that (7.7) holds everywhere on $K_{j}$. Since $g$ is absolutely continuous, it follows from (7.9) that $F^{\prime \prime}$ exists on components. If we differentiate (7.9) and use (7.7) we see that $F$ is quadratic on components and that (7.2) holds. This proves the lemma.

We emphasize that we have shown that on components $g$ is linear (relation (7.7)), $F$ is quadratic (relation (7.2)), and $z$ is quartic (relation (7.1)).

8. Structure of $\Omega$. In this section we shall determine the structure of $\Omega=$ $\{u: z(u) \neq 0\}$ and derive a useful integro-differential equation that $z$ satisfies.

LEMMA 4. There exists a real number $\lambda>0$ such that $z(u)=0$ for all $u \geqq \lambda$.

Suppose the assertion false. Then either there exists a component of $\Omega$ of the form $(a, \infty)$, or there exists an infinite number of components $\left(\alpha_{n}, \beta_{n}\right)$ tending to 
infinity. The first alternative cannot occur. For by Lemma $3, z$ is a quartic on $(a, \infty)$. Since $z$ is in $L_{1}[0, \infty]$, this is impossible.

To rule out the other possibility we proceed as follows. Define

$$
\psi(u)=e^{u} \int_{u}^{\infty} e^{-t} F(t) d t
$$

Then since $F$ is in $L_{2}$

$$
|\psi(u)|=e^{u}\left|\int_{u}^{\infty} e^{-t} F(t) d t\right| \leqq e^{u}\left(\int_{u}^{\infty} e^{-2 t} d t\right)^{1 / 2}\left(\int_{u}^{\infty} F^{2} d t\right)^{1 / 2}
$$

Thus $\psi(u)$ is defined for all $u$ and

$$
\psi(u)=o(1)
$$

as $u \rightarrow \infty$. Moreover, $\psi$ has an absolutely continuous derivative $\psi^{\prime}$. Differentiation of (8.1) yields

$$
\psi(u)-\psi^{\prime}(u)=F(u) \text { everywhere. }
$$

Differentiation of (8.3) yields

$$
\psi^{\prime}(u)-\psi^{\prime \prime}(u)=F^{\prime}(u) \text { a.e. }
$$

Upon differentiating (1.2) with $G$ replaced by $F$ and $y^{\prime}$ replaced by $z^{\prime}$, we get

$$
z^{\prime \prime}(u)=-F(u)-F^{\prime}(u) \text { a.e. }
$$

If we substitute (8.3) and (8.4) into this equation we get

$$
z^{\prime \prime}(u)=\psi^{\prime \prime}(u)-\psi(u) \text { a.e. }
$$

From the definition of $g$ in (6.7), from the definition of $\psi$ in (8.1), and from (8.3) we get

$$
g(u)=-F(u)+\psi(u)=\psi^{\prime}(u) \text { everywhere. }
$$

Hence

$$
\psi^{\prime \prime}(u)=g^{\prime}(u) \text { a.e. }
$$

For $u$ in $\Omega$, however, we have by (7.7) that $g^{\prime}(u)$ exists and is equal to $I \sigma(u)$. Hence $\psi^{\prime \prime}(u)$ exists and

$$
\psi^{\prime \prime}(u)=I \sigma(u), \quad u \in \Omega .
$$

Substituting this into (8.6) we see that

$$
z^{\prime \prime}(u)-I \sigma(u)=-\psi(u), \quad u \in \Omega .
$$

Since $\psi(u)=o(1)$ as $u \rightarrow \infty$, we now obtain

$$
\lim _{u \rightarrow \infty}\left[z^{\prime \prime}(u)-I \sigma(u)\right]=0,
$$

where the limit is taken over values of $u$ in $\Omega$. 
Let $\left(\alpha_{n}, \beta_{n}\right)$ be a sequence of components tending to infinity. It follows from (8.11) that on all but a finite number of these components

$$
\text { signum } z^{\prime \prime}(u)=\operatorname{signum} z(u)
$$

for all $u$ in the component. Since $z\left(\alpha_{n}\right)=z\left(\beta_{n}\right)=0$, this is impossible, and the lemma is proved.

We next derive an integro-differential equation that $z$ satisfies. This relation will enable us to obtain more precise information about the minimizing function $z$.

LEMMA 5. The minimizing function $z$ satisfies the following relation:

$$
I \int_{u}^{\infty}|z| d t+\int_{u}^{\infty} F^{2} d t=\chi(u)+F(u) z^{\prime}(u)+F^{2}(u) / 2, \quad 0 \leqq u<\infty
$$

where

$$
\begin{aligned}
\chi(u) & =0 & & \text { if } z(u)=0, \\
& =-z(u) F^{\prime}(u) & & \text { if } z(u) \neq 0 .
\end{aligned}
$$

We point out that (8.12) is the analogue of (8.4) in [2].

Suppose that $z(u) \neq 0$. Then $u$ is an interior point of a component $(\alpha, \beta)$, and

$$
I \int_{u}^{\infty}|z| d t=I \int_{u}^{\beta}|z| d t+I \int_{B}|z| d t
$$

where $B$ is the union of components $K_{j}=\left(\alpha_{j}, \beta_{j}\right)$ such that $\alpha_{j} \geqq \beta$. Hence, since (7.2) holds on components, since $F$ is quadratic on components, and since $z$ vanishes at the end points of components we obtain

$$
\begin{aligned}
I \int_{u}^{\infty}|z| d t & =I \int_{u}^{\beta} z \sigma d t+I \int_{B} z \sigma d t \\
& =\int_{u}^{\beta} z F^{\prime \prime} d t+\int_{B} z F^{\prime \prime} d t=\int_{u}^{\beta} z d F^{\prime}+\int_{B} z d F^{\prime} \\
& =-z(u) F^{\prime}(u)-\int_{u}^{\beta} z^{\prime} F^{\prime} d t-\int_{B} z^{\prime} F^{\prime} d t
\end{aligned}
$$

In Lemma 4 we established the existence of a real number $\lambda$ such that $z(v)=0$ for $v \geqq \lambda$. Hence

$$
\int_{u}^{\lambda} z^{\prime} F^{\prime} d t=\left(\int_{u}^{\beta}+\int_{B}+\int_{z}+\int_{N}\right) z^{\prime} F^{\prime} d t
$$

where

$$
\begin{aligned}
& Z=\left\{t: t \leqq \lambda, z(t)=0, z^{\prime}(t)=0\right\}, \\
& N=\left\{t: t \leqq \lambda, z(t)=0, z^{\prime}(t) \neq 0\right\} .
\end{aligned}
$$

The set $N$ is clearly isolated and hence of measure zero. Therefore,

$$
\int_{u}^{\lambda} z^{\prime} F^{\prime} d t=\left(\int_{u}^{\beta}+\int_{B}\right) z^{\prime} F^{\prime} d t
$$


Substituting this into (8.13) gives

$$
I \int_{u}^{\infty}|z| d t=-z(u) F^{\prime}(u)-\int_{u}^{\lambda} z^{\prime} F^{\prime} d t
$$

We have already shown that $z^{\prime}$ is absolutely continuous. Therefore, since $z(u)=0$ for $u \geqq \lambda$, we have $z^{\prime}(\lambda)=0$. Hence if we integrate by parts on the right in (8.14) and make use of (8.5) we get

$$
I \int_{u}^{\infty}|z| d t=-z(u) F^{\prime}(u)+z^{\prime}(u) F(u)+F^{2}(u) / 2-F^{2}(\lambda) / 2-\int_{u}^{\lambda} F^{2} d t
$$

From (8.5) it follows that $F(t)+F^{\prime}(t)=0$ for $t \geqq \lambda$, and so $F(t)=F(\lambda) e^{-(t-\lambda)}$, $t \geqq \lambda$. Hence

$$
\int_{\lambda}^{\infty} F^{2} d t=F^{2}(\lambda) / 2
$$

Using this relation in (8.15) gives

$$
I \int_{u}^{\infty}|z| d t+\int_{u}^{\infty} F^{2} d t=-z(u) F^{\prime}(u)+z^{\prime}(u) F(u)+F^{2}(u) / 2 .
$$

Thus we have established (8.12) for $u$ such that $z(u) \neq 0$.

If $z(u)=0$, then either $u$ is a limit point of $\Omega$ or $u$ is an interior point of an interval on which $z(t)=0$. In the first case, there exists a sequence of points $u_{n}$ in $\Omega$ such that $\lim _{n \rightarrow \infty} u_{n}=u$. For each $u_{n}$ the formula (8.12) holds. From (8.7) we have that for all $t$,

$$
F^{\prime}(t)=-g^{\prime}(t)+\psi^{\prime}(t)=-g^{\prime}(t)+g(t) .
$$

In the proof of the absolute continuity of $F$ we showed that $g^{\prime}$ satisfies (6.2). Thus $g^{\prime}$ is bounded. From the continuity of $g$ we get that $g$ is bounded in any neighborhood of $u$. Hence $F^{\prime}$ is bounded in any neighborhood of $u$. Therefore, if we let $u_{n} \rightarrow u$, we get (8.12) for the case in which $u$ is a boundary point of $\Omega$.

Suppose now that $u$ is an interior point of an interval on which $z$ vanishes. Then if $\beta \leqq \infty$ is the right-hand end point of this interval, we have

$$
I \int_{u}^{\infty}|z| d t+\int_{u}^{\infty} F^{2} d t=I \int_{\beta}^{\infty}|z| d t+\int_{\beta}^{\infty} F^{2} d t+\int_{u}^{\beta} F^{2} d t
$$

The first two integrals on the right fall under cases already established. Therefore, we can replace them by the right-hand side of (8.12). From the definition of $\beta$ and the continuity of $z$ and $z^{\prime}$ it follows that $z(\beta)=z^{\prime}(\beta)=0$, so that

$$
I \int_{u}^{\infty}|z| d t+\int_{u}^{\infty} F^{2} d t=F^{2}(\beta) / 2+\int_{u}^{\beta} F^{2} d t .
$$

On the interval $[u, \beta], z(t)=0$. Hence $z^{\prime \prime}(t)=0$ on this interval and from (8.6) we get that $F^{\prime}(t)+F(t)=0$ on $[u, \beta]$. Therefore, for $t$ in $[u, \beta]$,

$$
F(t)=F(u) e^{-(t-u)}
$$


and so

$$
\int_{u}^{\beta} F^{2} d t=F^{2}(u) / 2-F^{2}(\beta) / 2
$$

If we combine this relation with (8.17) we get

$$
I \int_{u}^{\infty}|z| d t+\int_{u}^{\infty} F^{2} d t=F^{2}(u) / 2
$$

Since $z(u)=z^{\prime}(u)=0$, this equation is (8.12) in the case under consideration. Lemma 5 is thus established.

LEMMA 6. If $z(\alpha)=0$ and $z^{\prime}(\alpha)=0$ for some $\alpha \geqq 0$, then $z(u)=0$ for all $u \geqq \alpha$.

From the definition of $m$ we get

$$
m=I^{2}+\int_{0}^{\infty} F^{2} d t=I \int_{0}^{\alpha}|z| d t+\int_{0}^{\alpha} F^{2} d t+I \int_{\alpha}^{\infty}|z| d t+\int_{\alpha}^{\infty} F^{2} d t .
$$

If we now apply (8.12) to the last two integrals and use the hypothesis $z(\alpha)=z^{\prime}(\alpha)=0$, we get

$$
\begin{aligned}
m & =I \int_{0}^{\alpha}|z| d t+\int_{0}^{\alpha} F^{2} d t+F^{2}(\alpha) / 2 \\
& =\left(\int_{0}^{\alpha}|z| d t\right)^{2}+\int_{0}^{\alpha} F^{2} d t+\left(\int_{\alpha}^{\infty}|z| d t\right)\left(\int_{0}^{\alpha}|z| d t\right)+F^{2}(\alpha) / 2 .
\end{aligned}
$$

Let $z_{0}$ be defined as follows

$$
\begin{aligned}
z_{0}(u) & =z(u) & & \text { if } u \leqq \alpha, \\
& =0 & & \text { if } u \geqq \alpha .
\end{aligned}
$$

Let $F_{0}$ be the function related to $z_{0}$. Then it follows from (1.2) that for $u \leqq \alpha$, $F_{0}(u)=F(u)$ and for $u \geqq \alpha$,

$$
F_{0}(u)=e^{-u}\left[1+\int_{0}^{\alpha} e^{t} z^{\prime}(t) d t\right]=e^{\alpha-u} F(\alpha) .
$$

Thus, $F_{0}$ is in $L_{2}[0, \infty]$ and $z_{0}$ is in $Y$. Hence

$$
J\left(z_{0}\right)=\left(\int_{0}^{\alpha}|z| d t\right)^{2}+\int_{0}^{\alpha} F^{2} d t+F^{2}(\alpha) / 2
$$

Comparing this with (8.18) we see that $J\left(z_{0}\right)<m$ unless $z(u)=0$ for $u \geqq \alpha$. Hence, since $z$ is minimizing we must have $z(u)=0$ for $u \geqq \alpha$.

Corollary 1. $z^{\prime}(0) \neq 0$.

If $z^{\prime}(0)=0$, then by the lemma we have that $z(u)=0$ for all $u$. From (1.2) we get that $F(u)=\exp (-u)$ for all $u$. Hence $J(z)=\frac{1}{2}$. 
Let $0<\alpha<\beta<\infty$ and let $y$ be any $C^{(1)}$ function that vanishes outside of $(\alpha, \beta)$ and does not vanish on $(\alpha, \beta)$. It follows from (8.12) that

$$
J(y)=G^{2}(\alpha) / 2+\int_{0}^{\alpha} G^{2} d t
$$

where $G$ is the function related to $y$. Since $y(u)=0$ on $0 \leqq u \leqq \alpha$, we get from (1.2) that $G(u)=\exp (-u)$ for $0 \leqq u \leqq \alpha$. It now follows from (8.19) that $J(y)=\frac{1}{2}$. Since the minimizing function is unique and $J(y)=J(z)=\frac{1}{2}$, it follows that $z$ cannot be the minimizing function. Hence $z^{\prime}(0)=0$ is impossible.

We point out that we have also proved by this argument that $m<\frac{1}{2}$.

LEMMA 7. $0<m<\frac{1}{2}$.

We have already shown that $m<\frac{1}{2}$. If $m=0$, then from the form of $J$ it is clear that $F \equiv 0$. But if $F \equiv 0$, then by (4.1) $z(u)=u$. This is not possible, since $z$ is in $L_{1}[0, \infty]$.

Lemma 6 and its corollary provide us with further information about $z$ and the structure of $\Omega$ as follows. From the corollary and the continuity of $z^{\prime}$ it follows that $\Omega$ has a component, say $K_{1}$, whose left-hand end point is $\alpha_{0}=0$. Since $z$ is in $L_{1}[0, \infty]$ and $z$ is a quartic on components, it follows that $K_{1}$ is finite. Let $\alpha_{1}$ denote its right-hand end point. Thus $z\left(\alpha_{1}\right)=0$. If $z^{\prime}\left(\alpha_{1}\right)=0$, then by Lemma 6 $z(u)=0$ for all $u \geqq \alpha_{1}$. If $z^{\prime}\left(\alpha_{1}\right) \neq 0$, then there is a component $K_{2}$ with left-hand end point at $\alpha_{1}$ and finite right-hand end point $\alpha_{2}$. Since $z$ does not vanish on $K_{1}$, the signs of $z^{\prime}\left(\alpha_{1}\right)$ and $z^{\prime}\left(\alpha_{0}\right)$ are opposite. Hence $z$ has opposite signs on $K_{1}$ and $K_{2}$.

By repeating the argument just made we see that either $z(u)=0$ for $u \geqq \alpha_{2}$ or there is a component $K_{3}$ on which the sign of $z$ is opposite to its sign on $K_{2}$. Proceeding inductively we see that $\Omega$ has one of two possible structures. Either $\Omega$ consists of a finite number of contiguous components of finite length or of an infinite number of contiguous components, each of finite length. Moreover, the sign of $z$ is different on contiguous components. We shall now show that it is not possible for $\Omega$ to have infinitely many components.

Suppose that $\Omega$ had an infinite number of components $K_{j}=\left(\alpha_{j}, \beta_{j}\right), j=1,2,3, \ldots$ Then by Lemma 4 , these components cannot go off to infinity; that is, there exists a real number $\lambda$ such that $\alpha_{n} \rightarrow \lambda, \beta_{n} \rightarrow \lambda$. The function $\psi$ defined in (8.1) is continuous. Therefore, from (8.11) we get that

$$
\lim _{u \rightarrow \lambda}\left[z^{\prime \prime}(u)-I \sigma(u)\right]=-\psi(\lambda),
$$

where the limit is taken through values of $u$ in $\Omega$.

Suppose first that $\psi(\lambda) \leqq 0$. Let $P$ denote the set of components on which $z(u)>0$. Since $z$ alternates in sign on contiguous components, every neighborhood of $\lambda$ contains infinitely many components of $P$. Since $\sigma(u)=1$ for $u$ in $P$, it follows from (8.20) that for infinitely many components belonging to $P, z^{\prime \prime}(u)>0$ and $z(u)>0$ for all $u$ in the component. This is clearly impossible since $z$ vanishes at the end points of a component. 
If $\psi(\lambda)>0$ we consider $N$, the collection of components on which $z(u)<0$. Then for $u$ in $N, \sigma(u)=-1$. Hence for infinitely many components belonging to $N$, $z^{\prime \prime}(u)<0$ and $z(u)<0$ for all $u$ in the component. This again is impossible.

We summarize the results of the discussion in the following lemma.

LEMMA 8. $\Omega$ consists of a finite number of contiguous components $\left(\alpha_{n}, \alpha_{n+1}\right)$, $n=0,1, \ldots, N-1$, where $\alpha_{0}=0$ and $\alpha_{N}=\lambda<\infty$. The function $z$ has opposite signs on contiguous components and $z^{\prime}\left(\alpha_{n}\right) \neq 0, n=0,1, \ldots, N-1$.

9. Completion of proof of Theorem 2. In this section we shall complete the proof of Theorem 2. We first summarize what has already been shown and what remains to be shown. Statement (i) of the Theorem was established in \$6. If we can establish that $z(u)>0$ on the component $K_{1}=\left(0, \alpha_{1}\right)$, then all of the statements of (ii), with the exception of (5.4) will follow from Lemma 3 and Lemma 8. Equation (5.4), however, is an immediate consequence of (8.5) and the fact that $z(u)=0$ for $u \geqq \lambda$. Thus to prove the theorem we must establish that $z(u)>0$ on $\left(\alpha_{0}, \alpha_{1}\right)$ and that (iii), (iv), and (v) hold.

We first establish (v). If we set $u=0$ in (8.12) we get

$$
m=F(0) z^{\prime}(0)+F^{2}(0) / 2 \text {. }
$$

From (1.2) we get

$$
F(0)=z^{\prime}(0)+1
$$

and hence $m=F(0)-F^{2}(0) / 2$, which is (5.7).

To establish (5.8) we consider the function $y(u ; a)=a z(u)$, where $a$ is any real number. It follows from (3.10) that the function $G_{a}$ related to $y(t ; a)$ is given by

$$
G_{a}(u)=a F(u)+(1-a) e^{-u} .
$$

Hence, for all $a, y(u ; a)$ is in $Y$ and

$$
\begin{aligned}
\varphi(a) & =\left(\int_{0}^{\infty}|a z| d t\right)^{2}+\int_{0}^{\infty}\left(a F+(1-a) e^{-t}\right)^{2} d t \\
& =a^{2} I^{2}+a^{2} \int_{0}^{\infty} F^{2} d t+2 a(1-a) \int_{0}^{\infty} F(t) e^{-t} d t+(1-a)^{2} \int_{0}^{\infty} e^{-2 t} d t
\end{aligned}
$$

has a minimum at $a=1$. Clearly, $\varphi^{\prime}(1)=0$. If we differentiate with respect to $a$, set $a=1$, and then set $\varphi^{\prime}(1)=0$ we get (5.8).

We next establish (iv). From (8.9), (8.7), and (8.6) we see that $z^{\prime \prime}$ is differentiable on components and that $z^{\prime \prime}(u)=-\psi^{\prime}(u)=g(u)$ on components. Since $g$ is continuous it follows that (5.6) holds. From (8.6) and (8.9) we get that on components

$$
z^{\prime \prime}(u)-I \sigma(u)=-\psi(u) .
$$

Since $\psi$ is continuous, we obtain (5.5). 
We next show that $z(u)>0$ on $\left(\alpha_{0}, \alpha_{1}\right)$. As already pointed out, this will establish (ii). The desired result will be a corollary of the following lemma.

Lemma 9. For each $n=0,1,2, \ldots, N-1, z^{\prime}\left(\alpha_{n}\right) z^{\prime \prime}\left(\alpha_{n}+0\right) \leqq 0$.

Suppose that the assertion is false for $n=i$. For the sake of definiteness, suppose that $z^{\prime}\left(\alpha_{i}\right)<0$ and $z^{\prime \prime}\left(\alpha_{i}+0\right)<0$. Since $z^{\prime}\left(\alpha_{i}\right)<0$ it follows that $z(u)<0$ for all $u$ in the component $\left(\alpha_{i}, \alpha_{i+1}\right)$. Hence, $z^{\prime \prime}(u)$ cannot be negative for all $u$ in $\left[\alpha_{i}, \alpha_{i+1}\right)$, and so $z^{\prime \prime}$ has at least one zero in $\left(\alpha_{i}, \alpha_{i+1}\right)$. But since $z(u)<0$ on $\left(\alpha_{i}, \alpha_{i+1}\right)$, we have from (7.1) that $\left(z^{\prime \prime}\right)^{\prime \prime}=z^{\mathrm{iv}}=I$ on $\left(\alpha_{i}, \alpha_{i+1}\right)$; i.e. the graph of $z^{\prime \prime}$ is concave up. Therefore $z^{\prime \prime}$ has precisely one zero, say $\gamma_{i}$ in $\left(\alpha_{i}, \alpha_{i+1}\right)$. Hence

$$
z^{\prime \prime}\left(\alpha_{i+1}-0\right)>0 \text {. }
$$

It now follows that $z^{\prime}$ decreases from $z^{\prime \prime}\left(\alpha_{i}\right)>0$ to its minimum value at $z^{\prime}\left(\gamma_{i}\right)$ and then increases on $\left(\gamma_{i}, \alpha_{i+1}\right)$. Since $z\left(\alpha_{i}\right)=z\left(\alpha_{i+1}\right)=0$, there is a point $\beta_{i}$ in $\left(\gamma_{i}, \alpha_{i+1}\right)$ such that $z^{\prime}(\beta)=0$. Hence since $z^{\prime \prime}(u)>0$ for $u$ in $\left(\gamma_{i}, \alpha_{i+1}\right)$, we have $z^{\prime}\left(\alpha_{i+1}\right)>0$.

Since $z^{\prime}\left(\alpha_{i+1}\right)>0$, there exists a component $\left(\alpha_{i+1}, \alpha_{i+2}\right)$ with $\alpha_{i+1}<\alpha_{i+2}<\infty$ on which $z(u)>0$. It now follows from (5.5) and (9.4) that

$$
z^{\prime \prime}\left(\alpha_{i+1}+0\right)=z^{\prime \prime}\left(\alpha_{i+1}-0\right)+2 I>0 .
$$

Thus $z^{\prime \prime}\left(\alpha_{i+1}+0\right) z^{\prime}\left(\alpha_{i+1}\right)>0$.

We can now repeat the argument and obtain the inequalities $z^{\prime \prime}\left(\alpha_{i+2}-0\right)<0$ and $z^{\prime}\left(\alpha_{i+2}\right)<0$. Hence there is a component $\left(\alpha_{i+2}, \alpha_{i+3}\right)$ contiguous to $\left(\alpha_{i+1}, \alpha_{i+2}\right)$. Proceeding inductively in this fashion we obtain the existence of infinitely many components. This contradicts Lemma 8, and so the present lemma is proved.

Corollary 1. $z(u)>0$ on $\left(0, \alpha_{1}\right)$.

Suppose that the corollary were false and $z(u)>0$ on $\left(0, \alpha_{1}\right)$. Then $z^{\prime}(0)<0$, since $z^{\prime}(0) \neq 0$. From (9.3), (8.1) and (5.8) we get that

$$
z^{\prime \prime}(0+)=I \sigma(0+)-\psi(0)=-I-m<0 .
$$

But by Lemma 9, we cannot have $z^{\prime \prime}(0+)$ and $z^{\prime}(0)<0$. Hence $z^{\prime}(0)>0$, and $z(u)>0$ on $\left(0, \alpha_{1}\right)$.

COROLlaRY 2. $z^{\prime \prime}(0+)=I-m<0$.

The equality follows from (9.3), (8.1), and (5.8). The inequality follows from the lemma and the fact that $z^{\prime}(0)>0$.

To complete the proof of the theorem we must prove (iii). From Lemma 6 we have that if $z\left(\alpha_{i}\right)=z^{\prime}\left(\alpha_{i}\right)=0$, then $z(u)=0$ for all $u \geqq \alpha_{i}$. Hence $z^{\prime}\left(\alpha_{i}\right) \neq 0$, except for $z^{\prime}\left(\alpha_{N}\right)$, which is equal to zero since $z(u)=0$ for all $u \geqq \alpha_{N}=\lambda$. Hence (iii) will be proved once we prove the following lemma.

LEMMA 10. In any component $\left(\alpha_{i}, \alpha_{i+1}\right), z^{\prime}$ has precisely one zero. 
For the sake of definiteness, suppose that $z(u)>0$ on $\left(\alpha_{i}, \alpha_{i+1}\right)$ and that $\left(\alpha_{i}, \alpha_{i+1}\right)$ is not the last component. Then $z^{\prime}\left(\alpha_{i}\right)>0$ and $z^{\prime}\left(\alpha_{i+1}\right)<0$. Since $z^{\prime}$ is a cubic on $\left(\alpha_{i}, \alpha_{i+1}\right)$ it either has one or three zeroes (counting multiplicities) on $\left(\alpha_{i}, \alpha_{i+1}\right)$. If the second alternative holds, then $z^{\prime \prime}=\left(z^{\prime}\right)^{\prime}$ has two zeroes on $\left(\alpha_{i}, \alpha_{i+1}\right)$. Since $z^{\prime}\left(\alpha_{i}\right)>0$, we must have, according to Lemma 9, $z^{\prime \prime}\left(\alpha_{i}+0\right)<0$. Since $z^{\prime \prime}$ has two zeroes on $\left(\alpha_{i}, \alpha_{i+1}\right)$, we must also have $z^{\prime \prime}\left(\alpha_{i+1}-0\right)<0$. Hence

$$
z^{\prime \prime}\left(\alpha_{i+1}+0\right)=z^{\prime \prime}\left(\alpha_{i+1}-0\right)-2 I<0 .
$$

Since $z^{\prime}\left(\alpha_{i+1}\right)<0$, this is impossible.

We leave the adjustments in the arguments to cover the case in which $\left(\alpha_{i}, \alpha_{i+1}\right)$ is the last interval, to the reader.

With this lemma the proof of Theorem 2 is completed.

\section{REFERENCES}

1. N. Wiener, Extrapolation, interpolation and smoothing of stationary time series, Wiley, New York, 1950.

2. L. D. Berkovitz and H. Pollard, A non-classical variational problem arising from an optimal filter problem, Arch. Rational Mech. Anal. 26 (1967), 281-304.

3. W. Rudin, Real and complex analysis, McGraw-Hill, New York, 1966.

4. N. I. Ahiezer, The calculus of variations, Blaisdell, New York, 1962.

Purdue University,

LAFAYETTE, INDIANA 